Bull. Soc. math. France

133 (3), 2005, p. 349-362

\title{
ON THE PYTHAGORAS NUMBERS OF REAL ANALYTIC SET GERMS
}

\author{
By José F. Fernando \& Jesús M. Ruiz
}

\begin{abstract}
We show that (i) the Pythagoras number of a real analytic set germ is the supremum of the Pythagoras numbers of the curve germs it contains, and (ii) every real analytic curve germ is contained in a real analytic surface germ with the same Pythagoras number (or Pythagoras number 2 if the curve is Pythagorean). This gives new examples and counterexamples concerning sums of squares and positive semidefinite analytic function germs.

RÉSUMÉ (Sur le nombre de Pythagore des germes d'ensembles analytiques réels)

Nous montrons : (i) que le nombre de Pythagore d'un germe d'ensemble analytique réel est le plus grand des nombres de Pythagore des courbes qu'il contient et (ii) que tout germe de courbe analytique réelle est contenu dans le germe d'une surface analytique réelle ayant le même nombre de Pythagore (ou le nombre 2 si la courbe est pythagoricienne). Cela fournit de nouveaux exemples et contre-exemples à propos des sommes de carrés et des germes de fonctions analytiques semi-définies.
\end{abstract}

\section{Preliminaries and statement of results}

The Pythagoras number of a ring $A$ is the smallest integer $p(A)=p \geq 1$ such that any sum of squares of $A$ is a sum of $p$ squares, and $p(A)=+\infty$ if such an

Texte reçu le 23 avril 2003, accepté le 3 février 2004

José F. Fernando, Depto. Matemáticas, F. Ciencias, Univ. Autónoma de Madrid, 28049 Madrid (Spain) • E-mail : josefrancisco.fernando@uam.es.

Jesús M. Ruiz, Depto. Geometría y Topología, F. Ciencias Matemáticas, Univ. Complutense de Madrid, 28040 Madrid (Spain) • E-mail : jesusr@mat.ucm.es.

2000 Mathematics Subject Classification. - 14P15.

Key words and phrases. - Pythagoras number, sum of squares, M. Artin's approximation. Supported by Spanish GAAR BFM2002-04797 and European RAAG HPRN-CT-2001-00271. 
integer does not exist. This invariant appeals specialists from many different areas, and has a very interesting behaviour in geometric cases; we refer the reader to [4], [7], [21] and [22]. Here we are interested in the important case of real analytic germs, which have been extensively studied in [6], [17], [18], [11], [20], [12], [8], [9], [10].

Let $X \subset \mathbb{R}^{n}$ be a real analytic set germ and $\mathcal{O}(X)$ its ring of analytic function germs. Since $\mathcal{O}\left(\mathbb{R}^{n}\right)$ is the ring $\mathbb{R}\{x\}$ of convergent power series in $x=\left(x_{1}, \ldots, x_{n}\right)$, we have $\mathcal{O}(X)=\mathbb{R}\{x\} / \mathcal{J}(X)$, where $\mathcal{J}(X)$ stands for the ideal of analytic function germs vanishing on $X$. We will discuss the Pythagoras number $p[X]=p(A)$ of the $\operatorname{ring} A=\mathcal{O}(X)$.

Clearly, if we have another real analytic set germ $Y \subset X$, then $\mathcal{J}(Y) \supset \mathcal{J}(X)$ and the canonical surjection $\mathcal{O}(X) \rightarrow \mathcal{O}(Y)$ gives immediately the inequality $p[Y] \leq p[X]$. This easy remark can be sharpened as follows:

TheOREM 1.1. - The Pythagoras number $p[X]$ of a real analytic set germ $X$ $i s$ the supremum of the Pythagoras numbers $p[Y]$ of all real analytic curve germs $Y \subset X$.

If the germ $X$ is irreducible, the supremum can be restricted to irreducible curve germs $Y$. In general this is not possible:

EXAmple 1.2. - The planar curve germ $Y:\left(x^{2}-y^{3}\right)\left(x^{2}+y^{3}\right)=0$ has Pythagoras number 2, while its irreducible components $Y_{1}: x^{2}-y^{3}=0$ and $Y_{2}: x^{2}+y^{3}=0$ have both Pythagoras number 1 .

Indeed, since $Y \subset \mathbb{R}^{2}$, we have $p[Y] \leq 2$, and looking at the initial forms of the series involved, one easily checks that $x^{2}+y^{2}$ is not a square mod $\left(x^{2}-y^{3}\right)\left(x^{2}+y^{3}\right)$. On the other hand, $\mathcal{O}\left(Y_{i}\right) \equiv \mathbb{R}\left\{t^{2}, t^{3}\right\}$, and this ring consists of all power series without the degree 1 monomial; it follows readily that in this ring every sum of squares has always a square root.

Note that if $X$ itself is a curve germ, Theorem 1.1 is trivial. On the other hand, in the irreducible case the result is quite more precise, as it takes the form of a curve selection lemma:

THEOREM 1.3. - Let $X$ be an irreducible real analytic set germ of dimension $\geq 2$, and $Z \subset X$ a semianalytic germ with $\operatorname{dim}(Z)=\operatorname{dim}(X)$. Then $p[X]$ is the supremum of the Pythagoras numbers $p[Y]$ of all irreducible curve germs $Y$ such that $Y \backslash\{0\} \subset Z$.

The condition $Y \backslash\{0\} \subset Z$ means that $Z$ contains both open half-branches of $Y$, which improves the more typical one half-branch selection; this is important for applications (see $[2$, VII.4,5]). Summing up, there are two possibilities:

(i) If $\operatorname{dim}(X)=2$, then $p[X]=p<+\infty$ (see [8]), and we can find a curve germ $Y \subset X$, with $p[Y]=p$. As said above, if $X$ is irreducible, the curve germ $Y$ can be chosen irreducible and anywhere in $X$.

TOME $133-2005-\mathrm{N}^{\mathrm{O}} 3$ 
(ii) If $\operatorname{dim}(X) \geq 3$, then $p[X]=+\infty$ (see [9]), and what happens is that $X$ contains anywhere irreducible curve germs with Pythagoras number arbitrarily large (note that in this case we can always suppose $X$ irreducible). For instance, in $X=\mathbb{R}^{3}$ we can find monomial curve germs $Y: x_{i}=t^{m_{i}}$ with $p[Y] \rightarrow+\infty$.

In this statements, anywhere means in any semianalytic set germ of maximal dimension. We will prove this in Section 2.

In case (ii) we can also find irreducible surface germs $X^{\prime} \subset X$ with arbitrarily large Pythagoras number $p\left[X^{\prime}\right]$. For that, we first find irreducible curve germs $Y_{k} \subset X$ not contained in the singular locus of $X$ such that $p\left[Y_{k}\right] \rightarrow+\infty$; then, by the condition on the singular locus, $X$ contains some irreducible surface germ $X_{k} \supset Y_{k}$ (see [2, VII.5.1,VIII.2.5]), so that $p\left[X_{k}\right] \geq p\left[Y_{k}\right]$.

After these results it is only natural to seek for a converse, namely:

THEOREM 1.4. - Let $Y$ be a curve germ. Then there exist a pure surface germ $X \supset Y$ with

$$
p[X]= \begin{cases}2 & \text { if } p[Y]=1 \\ p[Y] & \text { otherwise }\end{cases}
$$

We call a set germ pure if its irreducible components have all the same dimension. In fact, if $Y$ is irreducible, $X$ can be found irreducible too. The statement above is the best possible one, since surface germs have Pythagoras number $\geq 2$.

The proof of Theorem 1.4 is developed in Section 3. It is interesting to remark here that for irreducible $Y$, the surface $X$ one obtains is birational to $\mathbb{R}^{2}$, that is, it has a parametrization $x=x(s, t)$ that induces an isomorphism $\mathcal{M}(X) \rightarrow \mathbb{R}(\{s, t\})$ between the fields of meromorphic function germs. In particular, although the Pythagoras number $p[X]$ of the domain $\mathcal{O}(X)$ is arbitrary, the Pythagoras number $p(X)$ of its field of fractions $\mathcal{M}(X)$ is always 2: $p(X)=p(\mathcal{M}(X))=p(\mathbb{R}(\{s, t\}))=2$

The construction used to prove Theorem 1.4 can be extended to obtain the following relative version of the result: If a surface germ $X$ has some irreducible components of dimension 1 , then those components embed in a surface germ $X^{\prime}$ so that $p\left[X \cup X^{\prime}\right]=p[X]$. The proof of this technical generalization is most predictable, and will not be detailed here.

We also notice that the surface $X$ may well need bigger embedding dimension than the curve $Y$ :

EXAMPLE 1.5. - Consider the curve germ $Y \subset \mathbb{R}^{3}$ given by

$$
Y: x=t^{5}, y=t^{11}, z=t^{18} .
$$

The Pythagoras number of this curve germ is $p[Y]=2$, as one can see after some (not completely straightforward) work using some ideas in [17], [18] 
and [11]. Consequently, by Theorem 1.4, $Y$ is contained in some surface germ $X$ with $p[X]=2$, but no such a surface germ can be embedded in $\mathbb{R}^{3}$.

This can be proven by way of contradiction, as we sketch next. Suppose $X \subset \mathbb{R}^{3}$, defined by an equation $f(x, y, z)=0$ which must have order 2 (otherwise, $x^{2}+y^{2}+z^{2}$ would not be a sum of two squares mod $f$ ). As $f$ belongs to $\mathcal{J}(Y)=\left(z^{2}-y x^{5}, z y^{2}-x^{8}, y^{3}-x^{3} z\right)$, we can take

$$
f=z^{2}-y x^{5}+2 a\left(z y^{2}-x^{8}\right)+2 b\left(y^{3}-x^{3} z\right),
$$

with $a, b \in \mathbb{R}\{x, y\}$. By the Weiertrass Preparation Theorem, we factorize $f=U P$, where

$$
U \in \mathbb{R}\{x, y, z\} \text { is a unit, and } P=z^{2}+2 B(x, y) z+C(x, y) .
$$

A small computation gives

$$
\begin{gathered}
U(x, y, 0) C=f(x, y, 0) \in\left(y^{3}, x^{6}\right) \\
2 U(x, y, 0) B+\frac{\partial U}{\partial z}(x, y, 0) C=\frac{\partial f}{\partial z}(x, y, 0) \in\left(y^{2}, x^{3}\right)
\end{gathered}
$$

and after the change of coordinates $v=z+B, f$ becomes $v^{2}+C-B^{2}$. Since $U$ is a unit, $U(x, y, 0)$ is a unit too, and we deduce

$$
C \in\left(y^{3}, x^{6}\right) \subset\left(y, x^{2}\right)^{3}, \quad B^{2} \in\left(y^{2}, x^{6}\right)^{2} \subset\left(y, x^{2}\right)^{3} .
$$

Thus our surface germ is now $X: v^{2}=F$, where $F=B^{2}-C \in\left(y, x^{2}\right)^{3}$. This surface must be equivalent to some surface germ in the list given in [10], but standard singularity theory (see [14, 9.2.12-14]) tells that this is not possible.

Thus, we can only expect a mild control on the embedding dimension of $X$. In fact, a careful analysis of the construction in Section 3 will give some bound for that embedding dimension in terms of numerical invariants of $Y$. For instance, if $Y$ is the curve of the example above, the embedding dimension of $X$ can be lowered to 18 !

An interesting consequence of Theorem 1.4 is this:

Corollary 1.6. - Every integer $p \geq 2$ is the Pythagoras number of a real analytic surface germ.

Proof. - Indeed, $p$ is the Pythagoras number of some curve germ by [17], and then 1.4 applies.

We can look more closely at the construction of these surface germs, which give new examples concerning the problem whether every positive semidefinite analytic function germ is a sum of squares of analytic function germs (in short, psd $=$ sos). In fact, given a curve $Y$ with Pythagoras number $p \geq 2$, the surface $X$ with $p[X]=p$ lies in a sandwich $Y \equiv Y \times\{0\} \subset X \subset Y \times \mathbb{R}^{d}$ for suitable $d \geq 1$. Thus:

TOME $133-2005-\mathrm{N}^{\mathrm{O}} 3$ 
(1) Any positive semidefinite function germ on $Y$, extends to a positive semidefinite function germ on $X$, and

(2) Every sum of squares on $X$ restricts to a sum of squares on $Y$.

Consequently, since psd $\neq \operatorname{sos}$ for $Y$, we conclude psd $\neq$ sos for $X$. In particular, choosing $Y$ with $p[Y]=2$, we produce a full range of new examples of real analytic surface germs $X$ with minimal Pythagoras number and psd $\neq$ sos. These examples include those in [10, Ex.1.5], which correspond to the simplest possible $Y$ 's: the planar curves $x^{n}=y^{n+1}$.

\section{Proof of Theorems 1.1 and 1.3}

The key result to these theorems is a curve selection lemma with large tangent space, which refines [3, Prop. 1] in various ways to fit our situation. We present a different proof which simplifies that of [3] and gives the generalization needed here:

LEMma 2.1. - Let $X \subset \mathbb{R}^{n}$ be a real analytic irreducible germ of dimension $\geq 2$, and $Z \subset X$ a semianalytic germ with $\operatorname{dim}(Z)=\operatorname{dim}(X)$. Then, for every integer $k \geq 1$ there is a real analytic curve germ $Y \subset X$ such that:

$$
Y \backslash\{0\} \subset Z \quad \text { and } \quad \mathcal{J}(Y) \subset \mathcal{J}(X)+\left(x_{1}, \ldots, x_{n}\right)^{k} .
$$

Proof. - For the proof, we can suppose

$$
Z=\left\{f_{1}>0, \ldots, f_{r}>0\right\} \cap X,
$$

where $f_{1}, \ldots, f_{r} \in \mathbb{R}\{x\}$. Since $X$ is irreducible, the ring $A=\mathcal{O}(X)=$ $\mathbb{R}\{x\} / \mathcal{J}(X)$ is a domain, whose quotient field we denote by $K$. By the hypothesis on the dimension of $Z$, there is a total ordering $\alpha$ of $K$ such that $f_{1}(\alpha)>0, \ldots, f_{r}(\alpha)>0$. Write $(x)=\left(x_{1}, \ldots, x_{n}\right)$ and let $\mathfrak{m}=(x) \bmod \mathcal{J}(X)$ be the maximal ideal of $A$. We consider the convex hull $V$ of $\mathbb{R}$ in $K$ with respect to $\alpha$ (see [2, II.3.6]): $V$ is a valuation $\operatorname{ring}$ of $K$ with residue field $\mathbb{R}$, and since $A$ is henselian, $V$ dominates $A$. Now, by Hironaka's resolution of singularities (see [13]), there is a finitely generated regular $A$-algebra $A^{\prime}$ such that $f=f_{1} \cdots f_{r}$ has only normal crossings in $A^{\prime}$. Furthermore, $A^{\prime}$ is proper over $A$, that is, if a valuation ring of $K$ contains $A$, then it contains $A^{\prime}$. Consequently, our valuation ring $V$ dominates some localization $B$ of $A^{\prime}$; let $\mathfrak{n}$ denote the maximal ideal of $B$. By Zariski's Subspace Theorem (see $[1,10.6]), A$ is a subspace of $B$ (with respect to the adic topologies); consequently:

$$
\text { There is an integer } \ell \geq 1 \text { such that } \mathfrak{n}^{\ell} \cap A \subset \mathfrak{m}^{k} .
$$

Note also that, since the residue fields of $A$ and $V$ are both $\mathbb{R}$, the residue field of $B$ is also $\mathbb{R}$. By the normal crossings condition on $f$, we can write

$$
f_{j}=u_{j} y_{1}^{m_{j 1}} \cdots y_{d}^{m_{j d}}
$$

BULletin DE LA SOCIÉtÉ MATHÉMATIQUE DE FRANCE 
where $y_{1}, \ldots y_{d}$, form a regular system of parameters of $B, d=\operatorname{dim}(A) \geq 2$, and $u_{j}$ is a unit, whose residue class $\bmod \mathfrak{n}$ we denote by $c_{j} \in \mathbb{R}$. Replacing $y_{j}$ by $-y_{j}$ if needed, we can suppose $y_{j}(\alpha)>0$, and since $f_{j}(\alpha)>0$, and $V$ dominates $B$, it follows $c_{j}>0$. We are to define a local homomorphism:

$$
\varphi: A \subset B \subset \widehat{B}=\mathbb{R}[[y]] \longrightarrow \mathbb{R}[[t]], \quad y=\left(y_{1}, \ldots, y_{d}\right)
$$

by a suitable choice $y_{i} \mapsto y_{i}(t)=t^{2 q_{i}}+a_{i} t^{e}$.

First of all, we choose exponents $q_{1}<\cdots<q_{d}$ so that all formal power series $h(y) \in \mathbb{R}[[y]]$ such that $h\left(t^{q_{1}}, \ldots, t^{q_{d}}\right)=0$ have order $\geq \ell$ (see $[3$, Lemma 2$]$ or $[8$, 5.1]). We claim that:

$$
\left\{\begin{array}{l}
\text { There is } e_{0} \geq q_{1}, \ldots, q_{d} \text { such that if } h\left(t^{2 q_{1}}, \ldots, t^{2 q_{d}}\right) \text { has order } \geq e_{0}, \\
\text { then } h(y) \text { has order } \geq \ell .
\end{array}\right.
$$

Indeed, consider the homomorphism $\psi: \mathbb{R}[[y]] \rightarrow \mathbb{R}[[t]]: h \mapsto h\left(t^{2 q_{1}}, \ldots, t^{2 q_{d}}\right)$, and its kernel $\mathfrak{q}$. The choice of the $q_{i}$ 's was made to have $\mathfrak{q} \subset(y)^{\ell}$. Now we consider the descending chain of ideals $\mathfrak{a}_{e}=\psi^{-1}\left(t^{e} \mathbb{R}[[t]]\right)$. Clearly $\bigcap_{e} \mathfrak{a}_{e}=\mathfrak{q}$, hence by Chevalley's Theorem (see [24, VII.5 Thm.13, p.270]), we find $e_{0}$ such that $\mathfrak{a}_{e_{0}} \subset \mathfrak{q}+(y)^{\ell} \subset(y)^{\ell}$. This is what we claimed.

We will use the following reformulation:

$$
\left\{\begin{array}{l}
\text { For any } a_{1}, \ldots, a_{d} \in \mathbb{R} \text { and } e \geq e_{0}, \text { if } h\left(t^{2 q_{1}}+a_{1} t^{e}, \ldots, t^{2 q_{d}}+a_{d} t^{e}\right) \\
\text { has order } \geq e_{0}, \text { then } h(y) \text { has order } \geq \ell
\end{array}\right.
$$

This follows readily from $(2.1 .2)$, because

$$
h\left(t^{2 q_{1}}, \ldots, t^{2 q_{d}}\right)=h\left(t^{2 q_{1}}+a_{1} t^{e}, \ldots, t^{2 q_{d}}+a_{d} t^{e}\right) \bmod t^{e} .
$$

Next, consider $y_{1}^{q_{2}}-y_{2}^{q_{1}} \in B \subset K$. Since $K$ is the quotient field of $A$, we find $g \in A, g \neq 0$, such that $g^{2}\left(y_{1}^{q_{2}}-y_{2}^{q_{1}}\right) \in A$. Then:

$$
\left\{\begin{array}{l}
\text { There are non zero real numbers } a_{1}, \ldots, a_{d} \in \mathbb{R} \text { and an odd } \\
\text { integer } e \geq e_{0}, \text { such that } g\left(t^{2 q_{1}}+a_{1} t^{e}, \ldots, t^{2 q_{d}}+a_{d} t^{e}\right) \neq 0, \\
\text { say } g\left(t^{2 q_{1}}+a_{1} t^{e}, \ldots, t^{2 q_{d}}+a_{d} t^{e}\right)=a t^{m}+\cdots, a \neq 0 .
\end{array}\right.
$$

For, fix $a_{1}, \ldots, a_{d} \in \mathbb{R}$, and assume

$$
g\left(t^{2 q_{1}}+a_{1} t^{e}, \ldots, t^{2 q_{d}}+a_{d} t^{e}\right)=0
$$

for every odd integer $e \geq e_{0}$. We now borrow a trick from [16], involving the power series in two variables

$$
g^{*}(t, z)=g\left(t^{2 q_{1}}+a_{1} z, \ldots, t^{2 q_{d}}+a_{d} z\right) .
$$

By assumption, $g^{*}\left(t, t^{e}\right)=0$ for all odd $e \geq e_{0}$, which implies $g^{*}(t, z)=0$. Hence

$$
0=g^{*}(0, z)=g\left(a_{1} z, \ldots, a_{d} z\right)=z^{\omega} g_{\omega}\left(a_{1}, \ldots, a_{d}\right)+\cdots,
$$

where $g_{\omega} \neq 0$ is the initial form of $g$. Thus $g_{\omega}\left(a_{1}, \ldots, a_{d}\right)=0$. Consequently, it is enough to pick $\left(a_{1}, \ldots, a_{d}\right) \in \mathbb{R}^{d}$ such that $g_{\omega}\left(a_{1}, \ldots, a_{d}\right) \neq 0$ and $a_{1} \neq 0, \ldots, a_{d} \neq 0$. This settles (2.1.4). 
After the preceding preparation we have the homomorphism

$$
\varphi: \mathbb{R}\{x\} \rightarrow B \subset \mathbb{R}[[y]] \longrightarrow \mathbb{R}[[t]], \quad y_{i} \longmapsto y_{i}(t)=t^{2 q_{i}}+a_{i} t^{e} ;
$$

write $y(t)=\left(y_{1}(t), \ldots, y_{d}(t)\right)$.

From (2.1.1) and (2.1.3) we deduce:

$$
\varphi^{-1}\left(t^{e} \mathbb{R}[[t]]\right) \subset \mathcal{J}(X)+(x)^{k} .
$$

On the other hand, by an easy computation with the equality

$$
\varphi\left(f_{j}\right)=\varphi\left(u_{j} y_{1}^{m_{j 1}} \cdots y_{d}^{m_{j d}}\right)=u_{j}(y(t)) y_{1}(t)^{m_{j 1}} \cdots y_{d}(t)^{m_{j d}}
$$

one gets

$$
\varphi\left(f_{j}\right)=c_{j} t^{2 m_{j}}+\cdots,
$$

where $c_{j}>0$ and $m_{j}=m_{j 1} q_{1}+\cdots+m_{j d} q_{d}$.

Similarly, using (2.1.4) one sees that:

$$
\varphi\left(g^{2}\left(y_{1}^{q_{2}}-y_{2}^{q_{1}}\right)\right)=a^{2} b t^{2 m+e^{\prime}}+\cdots,
$$

where $b=-a_{2} q_{1} \neq 0$ and $e^{\prime}=2 q_{2}\left(q_{1}-1\right)+e$ is odd.

The homomorphism $\varphi$ would be the curve germ we seek, if it were analytic. To amend that, we recall that any formal homomorphism $A \rightarrow \mathbb{R}[[t]]$ can be approximated (in the Krull topology) by analytic homomorphisms $A \rightarrow \mathbb{R}\{t\}$ (this is M. Artin's Approximation Theorem, see [23, III.5.1]). But a close enough approximation $A \rightarrow \mathbb{R}\{t\}$ induces a homomorphism $\widetilde{\varphi}: \mathbb{R}\{x\} \rightarrow \mathbb{R}\{t\}$, for which properties (2.1.5) to (2.1.7) hold true. Thus, we consider the real analytic curve germ $Y \subset X$ whose ideal is $\mathcal{J}(Y)=\operatorname{ker}(\widetilde{\varphi})$. First of all, this $Y$ verifies the large tangent space condition

$$
\mathcal{J}(Y) \subset \mathcal{J}(X)+(x)^{k}
$$

by (2.1.5). To check that $Y \backslash\{0\} \subset Z$, we consider the corresponding parametrization:

$$
t \longmapsto x(t)=\left(x_{1}(t), \ldots, x_{n}(t)\right)=\left(\widetilde{\varphi}\left(x_{1}\right), \ldots, \widetilde{\varphi}\left(x_{n}\right)\right) .
$$

Then (2.1.6) says that

$$
f_{j}\left(x_{1}(t), \ldots, x_{n}(t)\right)=\widetilde{\varphi}\left(f_{j}\right)=c_{j} t^{2 m_{j}}+\cdots>0
$$

both for $t>0$ and for $t<0$, so that the two half-branches of this parametrization lie in $Z=\left\{f_{1}>0, \ldots, f_{r}>0\right\} \cap X$. Consequently, the only thing that remains to check is that indeed these two half-branches are different, so that they truly parametrize $Y$. But exactly to this purpose we have the element $g^{2}\left(y_{1}^{q_{2}}-y_{2}^{q_{1}}\right) \in A$, say

$$
g^{2}\left(y_{1}^{q_{2}}-y_{2}^{q_{1}}\right)=h \bmod \mathcal{J}(X)
$$

with $h \in \mathbb{R}\{x\}$. By (2.1.7):

$$
h\left(x_{1}(t), \ldots, x_{n}(t)\right)=\widetilde{\varphi}(h)=\widetilde{\varphi}\left(g^{2}\left(y_{1}^{q_{2}}-y_{2}^{q_{1}}\right)\right)=a^{2} b t^{2 m+e^{\prime}}+\cdots,
$$

BULletin DE LA SOCIÉtÉ MATHÉMATIQUE DE FRANCE 
which changes sign from $t>0$ to $t<0$, because $b^{2} a \neq 0$ and $e^{\prime}$ is odd. This means that the two half-branches are different, and they parametrize completely $Y$. Thus the proof is finished.

Using the large tangent curve selection lemma 2.1 above, Theorems 1.1 and 1.3 follow by approximation.

Proof of Theorem 1.3. — We suppose $p[Y] \leq p$ for all irreducible curve germs $Y \subset X$ such that $\operatorname{dim}(Y \cap Z)=1$, and will see that $p[X] \leq p$. To that end, since $\mathcal{O}(X)=\mathbb{R}\{x\} / \mathcal{J}(X)$, we consider a power series $h \in \mathbb{R}\{x\}$ that is a sum of squares mod $\mathcal{J}(X)$. We pick generators $f_{1}, \ldots, f_{r}$, of the ideal $\mathcal{J}(X)$ and have to solve in $\mathbb{R}\{x\}$ the equation

$$
h=\mathrm{y}_{1}^{2}+\cdots+\mathrm{y}_{p}^{2}+\mathrm{z}_{1} f_{1}+\cdots+\mathbf{z}_{r} f_{r}
$$

By M. Artin's Approximation Theorem (see [15]), it is enough to find solutions $\bmod (x)^{k}$ for $k$ large, where $(x)=\left(x_{1}, \ldots, x_{n}\right)$ is the maximal ideal of $\mathbb{R}\{x\}$. We fix $k$ and proceed as follows.

By Lemma 2.1, there exists an irreducible curve germ $Y \subset X$ such that $\operatorname{dim}(Y \cap Z)=1$ and $\mathcal{J}(Y) \subset \mathcal{J}(X)+(x)^{k}$. Now, since $h$ is a sum of squares $\bmod \mathcal{J}(X)$, it is a sum of squares $\bmod \mathcal{J}(Y)$. But by assumption, $p[Y] \leq p$, so that there are $h_{1}, \ldots, h_{p} \in \mathbb{R}\{x\}, g \in \mathcal{J}(Y)$ such that

$$
h=h_{1}^{2}+\cdots+h_{p}^{2}+g .
$$

Then, as $\mathcal{J}(Y) \subset \mathcal{J}(X)+(x)^{k}$, we can write $g=g_{1} f_{1}+\cdots+g_{r} f_{r} \bmod (x)^{k}$, and consequently

$$
h=h_{1}^{2}+\cdots+h_{p}^{2}+g_{1} f_{1}+\cdots+g_{r} f_{r} \bmod (x)^{k} .
$$

This means that $(\star)$ has a solution $\bmod (x)^{k}$.

As was already said, it follows that $(\star)$ has a solution in $\mathbb{R}\{x\}$, and $h$ is a sum of squares mod $\mathcal{J}(X)$, as wanted.

Finally, a natural modification of the preceding argument settles the reducible case:

Proof of Theorem 1.1. - If $X$ is not irreducible, say $X=\bigcup_{i} X_{i}$, the argument follows the same pattern, once one remarks that if some $Y_{i} \subset X_{i}$ has a large enough tangent space, then $Y=\bigcup_{i} Y_{i}$ has large tangent space. This again, is an adic topologies matter. The key fact is this: Let $I, J \subset \mathbb{R}\{x\}$ be two ideals. Then for every $k \geq 1$ there is $\ell \geq 1$ such that

$$
\left(I+(x)^{\ell}\right) \cap\left(J+(x)^{\ell}\right) \subset I \cap J+(x)^{k} .
$$

The assertion is an instance of the Artin-Rees Lemma (see [5, III.3.1, Cor. 1]): just consider the canonical inclusion of finite $\mathbb{R}\{x\}$-modules

$$
F=A / I \cap J \longrightarrow E=A / I \times A / J,
$$

equiped with the $(x)$-adic topologies.

TOME $133-2005-\mathrm{N}^{\mathrm{O}} 3$ 


\section{Proof of Theorem 1.4}

Here we fix a real analytic curve germ $Y$, construct the surface $X$ of Theorem 1.4, and prove the following, from which the result follows:

$$
\text { If } p[Y] \leq q \text { where } q \geq 2 \text {, then } p[X] \leq q .
$$

To do that, we consider separately the irreducible case.

Proof of Theorem 1.4 for irreducible $Y$. - Let $Y \subset \mathbb{R}^{n}$ be an irreducible curve germ. Pick a parametrization

$$
t \longmapsto x(t)=\left(x_{1}(t), \ldots, x_{n}(t)\right),
$$

which induces an isomorphism

$$
\mathcal{O}(Y) \longrightarrow A=\mathbb{R}\{x(t)\}=\mathbb{R}\left\{x_{1}(t), \ldots, x_{n}(t)\right\} \subset \mathbb{R}\{t\} .
$$

Then, $\mathbb{R}\{t\} /(x(t))$ is a finite $\mathbb{R}$-linear space, say generated by $h_{1}(t) \in \mathbb{R}\{t\}, \ldots$, $h_{r}(t) \in \mathbb{R}\{t\}$. We will use the fact that $A$ contains all power series of large enough order, say of order $\geq d$.

After this preparation, we consider in $\mathbb{R}^{m}=\mathbb{R}^{n} \times \mathbb{R}^{d}$ the coordinates

$$
(x, y)=\left(x_{1}, \ldots, x_{n}, y_{0}, y_{1}, \ldots, y_{d-1}\right)
$$

and the parametrization

$$
(s, t) \longmapsto(x(t), y(s, t))=\left(x_{1}(t), \ldots, x_{n}(t), y_{0}(s, t), \ldots, y_{d-1}(s, t)\right),
$$

where $y_{j}(s, t)=s t^{j}$. This parametrization induces a finite homomorphism $\varphi: \mathbb{R}\{x, y\} \rightarrow \mathbb{R}\{s, t\}$. Indeed, let $(x, y)$ be the maximal ideal of $\mathbb{R}\{x, y\}$. Since $y_{0}(s, t)=s$, we have

$$
\mathbb{R}\{s, t\} /(x, y) \mathbb{R}\{s, t\}=\mathbb{R}\{s, t\} /(x(t), y(s, t))=\mathbb{R}\{t\} /(x(t)),
$$

and this module is a finite $\mathbb{R}$-vector space (generated by $h_{1}(t), \ldots, h_{r}(t)$ ). Hence $\mathbb{R}\{s, t\}$ is a finite $\mathbb{R}\{x, y\}$-module by Mather's Preparation Theorem (see [19, II.1.5]).

We consider the image of $\varphi$

$$
B=\varphi(\mathbb{R}\{x, y\})=\mathbb{R}\{x(t), y(s, t)\} \subset \mathbb{R}\{s, t\},
$$

its kernel $\mathfrak{p} \subset \mathbb{R}\{x, y\}$, and the corresponding isomorphism

$$
\mathbb{R}\{x, y\} / \mathfrak{p} \longrightarrow B=\mathbb{R}\{x(t), y(s, t)\} \subset \mathbb{R}\{s, t\} .
$$

Clearly, $A \subset B$, and the substitution $s=0$ gives a surjection $B \rightarrow A$.

Now note that, $\varphi$ being finite, $\operatorname{dim}(\mathbb{R}\{x, y\} / \mathfrak{p})=2$. Consequently, the zero set germ $X \subset \mathbb{R}^{m}$ of $\mathfrak{p}$ is an irreducible real analytic surface germ with $\mathcal{O}(X)=\mathbb{R}\{x, y\} / \mathfrak{p} \equiv B$; it is the analytic closure of the image of the parametrization $(s, t) \mapsto(x(t), y(s, t))$ (actually, $X$ is the image of that parametrization, but we do not need that here).

BULletin DE LA SOCiÉtÉ MATHÉMATIQUE DE FRANCE 
We see next that the inclusion $B \subset \mathbb{R}\{s, t\}$ is birational. In fact, we have the stronger property that

$$
s \mathbb{R}\{s, t\} \subset(y(s, t)) B \subset B .
$$

Indeed, since $h_{1}(t), \ldots, h_{r}(t)$ generate $\mathbb{R}\{s, t\} /(x, y) \mathbb{R}\{s, t\}$ over

$$
\mathbb{R}=\mathbb{R}\{x, y\} /(x, y),
$$

we deduce from Nakayama's lemma that

$$
\mathbb{R}\{s, t\}=\mathbb{R}\{x, y\} h_{1}+\cdots+\mathbb{R}\{x, y\} h_{r}=B h_{1}+\cdots+B h_{r} .
$$

Thus it suffices to see that $s h(t) \in(y(s, t)) B$ for every series $h \in \mathbb{R}\{t\}$. But if $h$ has order $\geq d$, then $h \in A \subset B$, and as $s=y_{0}(s, t)$, we are done. So we can argue by descending induction on the order $e<d$ of $h$ : write $h=a_{e} t^{e}+h^{\prime}(t)$, where $h^{\prime}$ has order $>e$, and then

$$
s h=a_{e} y_{e}(s, t)+s h^{\prime} \in(y(s, t)) B .
$$

Now, it is clear from the construction that $Y \equiv Y \times\{0\} \subset X \subset Y \times \mathbb{R}^{d}$, and it only remains to prove (3.1): given $q \geq 2$ such that $p[Y] \leq q$, we show that $p(B) \leq q$.

Let $f \in B$ be a sum of squares in $B$. Then $f$ is a sum of squares in $\mathbb{R}\{s, t\}$, hence a sum of two squares as follows:

$$
f=\left(s a_{1}(s, t)+b_{1}(t)\right)^{2}+\left(s a_{2}(s, t)+b_{2}(t)\right)^{2}, \quad a_{i} \in \mathbb{R}\{s, t\}, b_{i} \in \mathbb{R}\{t\} .
$$

Here we can assume that $b_{2}=0$, after writing

$\left(s a_{1}+b_{1}\right)^{2}+\left(s a_{2}+b_{2}\right)^{2}=\left(s \frac{a_{1} b_{1}+a_{2} b_{2}}{\sqrt{b_{1}^{2}+b_{2}^{2}}}+\sqrt{b_{1}^{2}+b_{2}^{2}}\right)^{2}+\left(s \frac{-a_{1} b_{2}+a_{2} b_{1}}{\sqrt{b_{1}^{2}+b_{2}^{2}}}\right)^{2}$, where $\sqrt{b_{1}^{2}+b_{2}^{2}} \in \mathbb{R}\{t\}$ and $b_{i} / \sqrt{b_{1}^{2}+b_{2}^{2}} \in \mathbb{R}\{t\}$.

If $b_{1}=0$, then $f=\left(s a_{1}\right)^{2}+\left(s a_{2}\right)^{2}$ is a sum of two squares in $B$ (recall $s \mathbb{R}\{s, t\} \subset B)$. Thus, we may suppose that $b_{1} \neq 0$.

Now, since the substitution $s=0$ gives a surjection $B \rightarrow A$ and $f$ is a sum of squares in $B$, then $b_{1}^{2}=f(0, t)$ is a sum of squares in $A$. As $p(A)=p[Y] \leq q$, we find power series $c_{1}, \ldots, c_{m}, d_{1}, \ldots, d_{m} \in A \subset \mathbb{R}\{t\}$ such that

$$
b_{1}^{2}=f(0, t)=c_{1}^{2}+d_{1}^{2}+\cdots+c_{m}^{2}+d_{m}^{2}, \quad 2 m= \begin{cases}q & \text { if } q \text { is even } \\ q+1, d_{m}=0 & \text { if } q \text { is odd }\end{cases}
$$

For later use, we rearrange the addends so that the orders of $b_{1}$ and $c_{1}$ coincide. An straightforward computation gives

$$
\begin{aligned}
f & =\left(s a_{1}+b_{1}\right)^{2}+\left(s a_{2}\right)^{2}=\left(\left(s a_{1}+b_{1}\right)^{2}+\left(s a_{2}\right)^{2}\right) \frac{1}{b_{1}^{2}} \sum_{j=1}^{m}\left(c_{j}^{2}+d_{j}^{2}\right) \\
& =\sum_{j=1}^{m}\left(s f_{j}+c_{j}\right)^{2}+\left(s g_{j}+d_{j}\right)^{2}
\end{aligned}
$$

TOME $133-2005-\mathrm{N}^{\mathrm{O}} 3$ 
where $f_{j}=\left(a_{1} c_{j}-a_{2} d_{j}\right) / b_{1}, g_{j}=\left(a_{1} d_{j}+a_{2} c_{j}\right) / b_{1} \in \mathbb{R}\{s, t\}$, because the order of $b_{1}$ is the minimum of the orders of the $c_{j}$ 's and the $b_{j}$ 's.

This is a representation of $f$ as a sum of $2 m$ squares in $B$ (recall that $s \mathbb{R}\{s, t\} \subset B$ and $c_{j}, d_{j} \in A \subset B$ ). If $q$ is even, then $2 m=q$ and we have finished. If $q$ is odd, then $2 m=q+1$ and some adjustment is required.

The adjustment will be to merge the last square into the first two. Note that if $q$ is odd then $q \geq 3, f_{m}=a_{1} c_{m} / b_{1}, g_{m}=a_{2} c_{m} / b_{1}$ and $d_{m}=0$. We consider the equation

$$
\left(s\left(f_{1}+\mathrm{y}\right)+c_{1}\right)^{2}+\left(s\left(g_{1}+\mathrm{z}\right)+d_{1}\right)^{2}=\left(s f_{1}+c_{1}\right)^{2}+\left(s g_{1}+d_{1}\right)^{2}+\left(s g_{m}\right)^{2} .
$$

If we solve it in $\mathbb{R}\{s, t\}$, we are done. Now, equating coefficients of the powers of $s$, we see it is enough to solve the following system

$$
\left\{\begin{array}{l}
P_{1}(\mathrm{y}, \mathrm{z})=2 \mathrm{y} f_{1}+\mathrm{y}^{2}+2 \mathrm{z} g_{1}+\mathrm{z}^{2}-\left(a_{2} \frac{c_{m}}{b_{1}}\right)^{2}=0, \\
P_{2}(\mathrm{y}, \mathrm{z})=\mathrm{y} \frac{c_{1}}{b_{1}}+\mathrm{z} \frac{d_{1}}{b_{1}}=0
\end{array}\right.
$$

where $c_{1} / b_{1} \in \mathbb{R}\{t\}$ and $d_{1} / b_{1} \in \mathbb{R}\{t\}$ (this apparently superfluous denominator $b_{1}$ will be essential soon!). The jacobian matrix of the previous system is

$$
\lambda(\mathrm{y}, \mathrm{z})=\left(\begin{array}{cc}
2 f_{1}+2 \mathrm{y} & 2 g_{1}+\mathrm{z} \\
c_{1} / b_{1} & d_{1} / b_{1}
\end{array}\right) .
$$

Let $I$ be the ideal of $\mathbb{R}\{s, t\}$ generated by

$$
\begin{aligned}
\operatorname{det} \lambda(0,0)= & 2\left(f_{1} \frac{d_{1}}{b_{1}}-g_{1} \frac{c_{1}}{b_{1}}\right) \\
& =2\left(\frac{a_{1} c_{1}-a_{2} d_{1}}{b_{1}} \cdot \frac{d_{1}}{b_{1}}-\frac{a_{1} d_{1}+a_{2} c_{1}}{b_{1}} \cdot \frac{c_{1}}{b_{1}}\right)=-2\left(\frac{c_{1}^{2}+d_{1}^{2}}{b_{1}^{2}}\right) a_{2} .
\end{aligned}
$$

Since $b_{1}$ and $c_{1}$ have the same order, $\left(c_{1}^{2}+d_{1}^{2}\right) / b_{1}^{2} \in \mathbb{R}\{t\}$ is a unit, and $I=\left(a_{2}\right)$. Moreover, $P_{1}(0,0)=-\left(a_{2} c_{m} / b_{1}\right)^{2} \in I^{2}$ and $P_{2}(0,0)=0 \in I^{2}$, so that we can apply Tougeron's Implicit Function Theorem (see [23, III.3.2]). Whence, the system has indeed a solution, as wanted.

Thus we have completed the proof of the case when $Y$ is irreducible. In fact we have proved the following more precise statement:

$$
\left\{\begin{array}{l}
\text { Let } f \in \mathbb{R}\{x, y\} \text { be a sum of squares on } X, \\
\text { with } f_{\mid Y}=\sum_{k=1}^{q} \beta_{k}^{2}, \beta_{k} \in \mathbb{R}\{x\} . \\
\text { Then there are } \alpha_{k} \in(y) \mathbb{R}\{x, y\} \text { such that } \\
f_{\mid X}=\sum_{k=1}^{q}\left(\alpha_{k}+\beta_{k}\right)^{2} .
\end{array}\right.
$$

Proof for $Y$ reducible. - By induction on the number of irreducible components we have a surface germ $X \subset \mathbb{R}^{n} \times \mathbb{R}^{d}$ with $Y \times\{0\} \subset X$ and the property (3.2). We are to find the same for $Y \cup Y^{\prime}, Y^{\prime}$ irreducible.

BULletin DE LA SOCiÉtÉ MATHÉmATiQUe DE FRANCE 
To start with, pick $X^{\prime} \subset \mathbb{R}^{n} \times \mathbb{R}^{d^{\prime}}$ with $Y^{\prime} \times\{0\} \subset X^{\prime}$ and the property (3.2), which now says:

If $f \in \mathbb{R}\left\{x, y^{\prime}\right\}$ is a sum of squares on $X^{\prime}$, with $f_{\mid Y^{\prime}}=\sum_{k=1}^{q} \beta_{k}^{\prime 2}$ and $\beta_{k}^{\prime} \in \mathbb{R}\{x\}$, then there are $\alpha_{k}^{\prime} \in\left(y^{\prime}\right) \mathbb{R}\left\{x, y^{\prime}\right\}$ such that

$$
\left.f^{\prime}\right|_{X^{\prime}}=\sum_{k=1}^{q}\left(\alpha_{k}^{\prime}+\beta_{k}^{\prime}\right)^{2}
$$

We consider $\mathbb{R}^{N}=\mathbb{R}^{n} \times \mathbb{R}^{d} \times \mathbb{R}^{d^{\prime}}$ and identify

$$
\mathbb{R}^{n} \times \mathbb{R}^{d} \equiv \mathbb{R}^{n} \times \mathbb{R}^{d} \times\{0\} \subset \mathbb{R}^{N}, \quad \mathbb{R}^{n} \times \mathbb{R}^{d^{\prime}} \equiv \mathbb{R}^{n} \times\{0\} \times \mathbb{R}^{d^{\prime}} \subset \mathbb{R}^{N},
$$

to have $Y \cup Y^{\prime} \subset X \cup X^{\prime} \subset \mathbb{R}^{N}$. We claim that $p[X] \leq q$.

Indeed, let $f \in \mathbb{R}\left\{x, y, y^{\prime}\right\}$ be a sum of squares on $X \cup X^{\prime}$. Then $f$ is a sum of squares on $Y \cup Y^{\prime}$, and can be written as a sum of $q$ squares, say

$$
f_{\mid Y \cup Y^{\prime}}=\sum_{k=1}^{q} g_{k}^{2}, \quad \text { where } g_{k} \in \mathbb{R}\{x\} .
$$

Now we look at $f_{\mid X}$ and using (3.2) write it as

$$
f_{\mid X}=\sum_{k=1}^{q}\left(\alpha_{k}+g_{k}\right)^{2}, \quad \text { where } \alpha_{k} \in(y) \mathbb{R}\{x, y\} .
$$

(note that $\left.f_{\mid Y}=\left(f_{\mid Y \cup Y^{\prime}}\right)_{\mid Y}\right)$. Similarly

$$
f^{\prime}{ }_{\mid X}^{\prime}=\sum_{k=1}^{q}\left(\alpha_{k}^{\prime}+g_{k}\right)^{2}, \quad \text { where } \alpha_{k}^{\prime} \in\left(y^{\prime}\right) \mathbb{R}\left\{x, y^{\prime}\right\} .
$$

Finally, we have

$$
f_{\mid X \cup X^{\prime}}=\sum_{k=1}^{q}\left(h_{k}+g_{k}\right)^{2}, \text { where } h_{k}=\alpha_{k}+\alpha_{k}^{\prime} \in\left(y, y^{\prime}\right) \mathbb{R}\left\{x, y, y^{\prime}\right\} .
$$

Indeed, it suffices to show that the sum on the right hand side coincides with $f$ on $X$ and on $X^{\prime}$. But by construction:

$$
\begin{array}{cc}
\alpha_{k \mid \mathbb{R}^{n} \times \mathbb{R}^{d} \times\{0\}}^{\prime} \equiv 0, & X \subset \mathbb{R}^{n} \times \mathbb{R}^{d} \times\{0\}, \\
\alpha_{k \mid \mathbb{R}^{n} \times\{0\} \times \mathbb{R}^{d^{\prime}}} \equiv 0, & X^{\prime} \subset \mathbb{R}^{n} \times\{0\} \times \mathbb{R}^{d^{\prime}} .
\end{array}
$$

This completes the induction.

\section{BIBLIOGRAPHY}

[1] AbHyankar (S.S.) - Resolution of singularities of embedded algebraic surfaces, 2nd, enlarged ed., Springer Monographs in Math., Springer Verlag, Berlin-Heidelberg-New York, 1998.

TOME $133-2005-\mathrm{N}^{\mathrm{O}} 3$ 
[2] Andradas (C.), Bröcker (L.) \& Ruiz (J.M.) - Constructible Sets in Real Geometry, Ergeb. Math. Grenzgeb., vol. 33, Springer Verlag, BerlinHeidelberg-New York, 1996.

[3] Becker (J.) \& Gurjar (R.) - Curves with large tangent space, Trans. Amer. Math. Soc., t. 242 (1975), pp. 285-296.

[4] Bochnak (J.), Coste (M.) \& Roy (M.-F.) - Real Algebraic Geometry, Ergeb. Math. Grenzgeb., vol. 36, Springer Verlag, Berlin-HeidelbergNew York, 1998.

[5] Bourbaki (N.) - Commutative Algebra, Hermann, Paris, 1972.

[6] Campillo (A.) \& Ruiz (J.M.) - Some Remarks on Pythagorean Real Curve Germs, J. Algebra, t. 128 (1990), pp. 271-275.

[7] Choi (M.D.), Dai (Z.D.), LAm (T.Y.) \& Reznick (B.) - The Pythagoras number of some affine algebras and local algebras, J. reine angew. Math., t. 336 (1982), pp. 45-82.

[8] Fernando (J.F.) - On the Pythagoras numbers of real analytic rings, J. Algebra, t. 243 (2001), pp. 321-338.

[9] _ Sums of squares in real analytic rings, Trans. Amer. Math. Soc., t. 354 (2002), pp. 1909-1919.

[10] _ Analytic surface germs with minimal Pythagoras number, Math. Z., t. 244 (2003), pp. 725-752.

[11] Fernando (J.F.) \& Quarez (R.) - Some remarks on the computation of Pythagoras numbers of real irreducible algebroid curves through Gram matrices, J. Algebra, t. 274 (2004), pp. 64-67.

[12] Fernando (J.F.) \& Ruiz (J.M.) - Positive semidefinite germs on the cone, Pacific J. Math., t. 205 (2002), pp. 109-118.

[13] Hironaka (H.) - Resolution of singularities of an algebraic variety over a field of characteristic zero, Ann. of Math., t. 79 (1964), pp. 109-123, 205-326.

[14] De Jong (T.) \& Pfister (G.) - Local Analytic Geometry, basic theory and applications, Advanced Lectures in Mathematics, Vieweg Verlag, Braunschweig-Wiesbaden, 2000.

[15] Kurke (H.), Mostowski (T.), Pfister (G.), Popescu (D.) \& Roczen (M.) - Die Approximationseigenschaft lokaler Ringe, Lect. Notes in Math., vol. 634, Springer Verlag, 1978.

[16] Merrien (J.) - Un théorème des zéros pour les idéaux de séries formelles à coefficients réels, C. R. Acad. Sci. Paris Sér. A-B, t. 276 (1973), pp. 10551058.

[17] Ortega (J.) - On the Pythagoras number of a real irreducible algebroid curve, Math. Ann., t. 289 (1991), pp. 111-123.

[18] Quarez (R.) - Pythagoras numbers of real algebroid curves and Gram matrices, J. Algebra, t. 238 (2001), pp. 139-158. 
[19] RuIZ (J.M.) - The basic theory of power series, Advanced Lectures in Mathematics, Vieweg Verlag, Braunschweig-Wiesbaden, 1993.

[20] Sums of two squares in analytic rings, Math. Z., t. 230 (1999), pp. $317-328$.

[21] SCHEIDERER (C.) - Sums of squares of regular functions on real algebraic varieties, Trans. Amer. Math. Soc., t. 352 (1999), pp. 1039-1069.

[22] _ On sums of squares in local rings, J. reine angew. Math., t. 540 (2001), pp. 205-227.

[23] Tougeron (J.-C.) - Idéaux de fonctions différentiables, Ergeb. Math. Grenzgeb., vol. 71, Springer Verlag, Berlin-Heidelberg-New York, 1972.

[24] Zariski (O.) \& Samuel (P.) - Commutative Algebra, II, Graduate Texts in Math., vol. 29, Springer Verlag, Berlin-Heidelberg-New York, 1980. 